\title{
A Nation-Wide Epidemiological Study of Newly Diagnosed Primary Spine Tumor in the Adult Korean Population, 2009-2011
}

\author{
Seil Sohn, M.D., Ph.D., ${ }^{1,2,3,4^{*}}$ Jinhee Kim, R.N., Ph.D., M.P.H., ${ }^{5 *}$ Chun Kee Chung, M.D., Ph.D., ${ }^{1,2,3,6}$ Na Rae Lee, B.S., ${ }^{7}$ \\ Moon Jun Sohn, M.D., Ph.D., ${ }^{8}$ Sung Hwan Kim, M.D., Ph.D. ${ }^{9}$ \\ Department of Neurosurgery, Seoul National University College of Medicine; Neuroscience Research Institute, ${ }^{2}$ Seoul National University \\ Medical Research Center; Clinical Research Institute, ${ }^{3}$ Seoul National University Hospital, Seoul; Department of Neurosurgery, ${ }^{4}$ HA Bundang \\ Medical Center, CHA University, Seongnam; Department of Nursing, ${ }^{5}$ College of Medicine, Chosun University, Gwangju; Department of Brain \\ and Cognitive Sciences, ${ }^{6}$ Seoul National University College of Natural Sciences, Seoul; National Evidence-based Healthcare Collaborating \\ Agency, Seoul; Department of Neurosurgery, Ilsan Paik Hospital, College of Medicine, Inje University, Goyang; Department of Radiation On- \\ cology, St. Vincent's Hospital, School of Medicine, The Catholic University of Korea, Suwon, Korea
}

Objective : This 2009-2011 nation-wide study of adult Koreans was aimed to provide characteristics, medical utilization states, and survival rates for newly diagnosed patients with primary nonmalignant and malignant spine tumors.

Methods : Data for patients with primary spine tumors were selected from the Korean Health Insurance Review and Assessment Service database. The data included their age, sex, health insurance type, co-morbidities, medical cost, and hospital stay duration. Hospital stay duration and medical costs per person occurring in one calendar year were used. In addition, survival rates of patients with primary malignant spine tumors were evaluated.

Results : The incidence rate of a primary spine tumor increased with age, and the year of diagnosis ( $p \leq 0.0001)$. Average annual medical costs ranged from 1627 USD (pelvis \& sacrum \& coccyx tumors) to 6601 USD (spinal cord tumor) for primary nonmalignant spine tumor and from 12137 USD (spinal meningomas) to 20825 USD (pelvis \& sacrum \& coccyx tumors) for a primary malignant spine tumor. Overall survival rates for those with a primary malignant spine tumor were $87.0 \%, 75.3 \%$, and $70.6 \%$ at 3,12 , and 24 months, respectively. The Cox regression model results showed that male sex, medicare insurance were significantly positive factors affecting survival after a diagnosis of primary malignant spine tumor.

Conclusion : Our study provides a detailed view of the characteristics, medical utilization states, and survival rates of patients newly diagnosed with primary spine tumors in Korea.

Key Words : Epidemiology · Spine · Neoplasms · Population.

- Received : May 31, 2016 • Revised : November 29, 2016 •Accepted : December 9, 2016

- Address for reprints : Chun Kee Chung, M.D., Ph.D.

Department of Neurosurgery, Seoul National University College of Medicine, 101 Daehak-ro, Jongno-gu, Seoul 03080, Korea

Tel : +82-2-2072-2350, Fax : +82-2-744-8459, E-mail : chungc@snu.ac.kr

*Seil Sohn and Jinhee Kim contributed equally to this work.

This is an Open Access article distributed under the terms of the Creative Commons Attribution Non-Commercial License (http://creativecommons.org/licenses/by-nc/4.0) which permits unrestricted non-commercial use, distribution, and reproduction in any medium, provided the original work is properly cited. 


\section{INTRODUCTION}

The relative rarity of primary malignant and nonmalignant spine tumors limits research, treatment decisions, and health care planning related to this condition ${ }^{2,4,5,10)}$. It is important to determine the epidemiology of primary spine tumor accurately for decision-making regarding treatment and for research and health care planning $3,6,13,15)$.

Population-based nation-wide studies are less subject to selection or non-response biases than case-series studies, thus their analyses can have high statistical power ${ }^{12}$. Korea has an obligatory health insurance system, and all medical reimbursement records for the entire Korean population are maintained in a single database that can be used to assess the nationwide effects of illnesses and consequent health care use $\mathrm{e}^{9,11,20)}$.

Several population-based incidence rates of spine tumor have been reported ${ }^{4,7,10,13,15,17)}$. Among these population-based studies, there have been 2 nation-wide studies in Korea ${ }^{7,17)}$. Such efforts could provide information to health care agencies and stimulate research on primary spine tumors. However, these 2 studies did not provide detailed investigations such as medical utilization states of each tumor type and survival analysis.

The aim of our nation-wide study of the adult Korean population was to ascertain the characteristics, medical utilization states, and survival rates of patients newly diagnosed with primary spine tumors.

\section{MATERIALS AND METHODS}

\section{Data source}

All Korean citizens are beneficiaries of the Korean National Health Insurance System ${ }^{8)}$, and nation-wide inpatient and outpatient data on disease and services (procedures and operations) are coded and registered in the Korean Health Insurance Review and Assessment Service (HIRA) database, thus enabling the undertaking of population-based studies". The HIRA database is a "fee-for-service" system. Disease codes used in the database are standardized according to the Korean Classification of Disease, 4th version, which follows the International Classification of Disease, 10th version (ICD-10) ${ }^{8)}$.

This study was approved by the institutional review boards at the National Evidence-based Healthcare Collaborating
Agency, Seoul National University Hospital, Korea Cancer Center Hospital, Ilsan Paik Hospital, and St. Vincent's Hospital.

\section{Patient population}

Patients over 20 years old with primary nonmalignant and malignant spine tumors as identified from the HIRA database between January 1, 2008 and December 31, 2012, were included in our study. A multidisciplinary panel consisting of spine surgeons (S.S., C.K.C, and M.J.S.), epidemiologists (J.H.K.) and a radiation oncologist (S.H.K) identified the patients with newly diagnosed primary spine tumors. Disagreements were resolved by consensus after a discussion.

A newly diagnosed nonmalignant and malignant spine tumor was defined by applying the following criteria; 1) ICD-10 codes for primary nonmalignant or primary malignant spine tumors, 2) magnetic resonance imaging of the spine within 1 year after the initial ICD-10 code assignment, 3) no previous identical tumor code history within 1 year, and 4) a hospital visit within 3 months after diagnosis (Table 1). The ICD-10 codes for primary malignant spine tumors were C41.2 (malignant neoplasm of the vertebral column), C41.4 (malignant neoplasm of the pelvic bones, sacrum and coccyx), C70.1 (malignant neoplasm of the spinal meninges), and C72.0 (malignant neoplasm of the spinal cord). The ICD-10 codes for primary nonmalignant spine tumors were D16.6 (benign neoplasm of the vertebral column), D16.8 (benign neoplasm of the pelvic bones, sacrum and coccyx), D32.1 (benign neoplasm of the spinal meninges), D33.4 (benign neoplasm of the spinal cord), and D42.1 (neoplasm of uncertain or unknown behavior of spinal meninges. Based on the above tumor definitions and on analyses of the patients' annual follow-up data, the population of newly diagnosed primary spine tumor patients in Korea during the period 2009-2011 was identified and included in this study.

Additionally, data regarding the patient's age, sex, health insurance type, medical cost, and hospital stay durations of each patient were obtained from the HIRA database.

For patients newly diagnosed with as primary nonmalignant and malignant spine tumors, the hospital stay duration and medical cost per person occurring within 1 calendar year following the diagnosis were included in our analysis.

In our assessment of survival rate according to each primary malignant spine tumor, we included patients newly diag- 
Epidemiology of Primary Spine Tumor | Sohn S, et al.

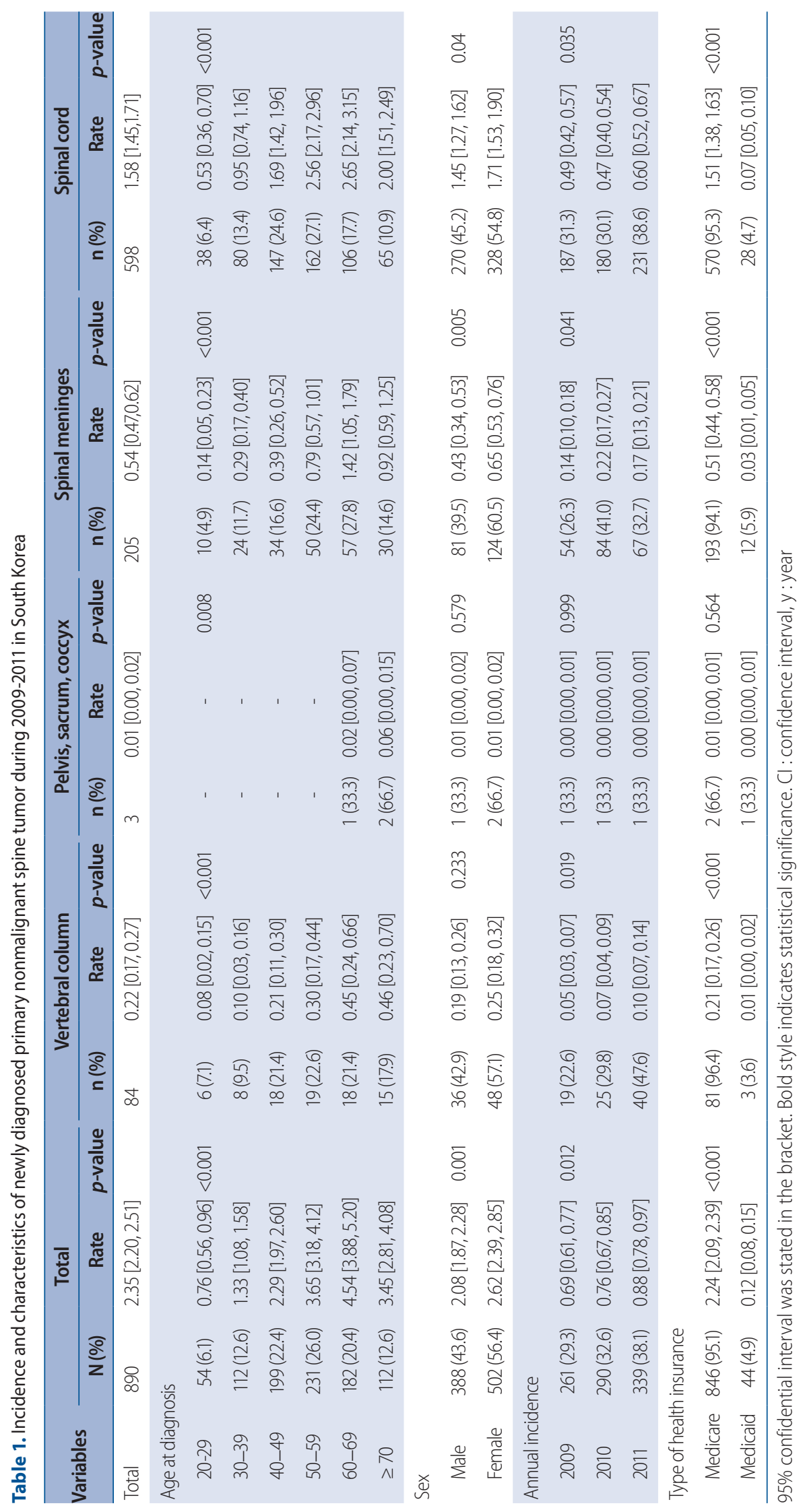


nosed with a primary malignant spine tumor in 2009. This allowed us to obtain 3 year follow-up data for the ensuing analysis. Comparative survival rates analyses were performed according to the 4 primary malignant spine tumors. Death was deemed to have occurred when patients with primary malignant spine tumor did not use any medical service for 6 consecutive months. We used this "death" definition after verifying that data from several duration other than 6 months produced similar results ${ }^{17)}$.

\section{Statistical analysis}

Overall incidence rates of primary nonmalignant and malignant spine tumors, as well as incidence rates by patient age at diagnosis, sex, diagnosis year, and health insurance type were calculated. All rates were age-adjusted and expressed as a number per 100000 persons. The age-adjusted rates were standardized to the "Age Structure of Population in Korea 2010" obtained from Statistics Korea. A Logistic regression analysis was performed to evaluate incidence rates by age and diagnosis year. Differences in incidence rates according to sex, and health insurance type were assessed by using chi-square tests. Survival rates in each primary malignant spine tumor were compared by using the Kaplan Meier method and Cox proportional hazard regressions. A two-tailed $p$ value $<0.05$ was considered indicative of a significant difference. For statistical analyses, SAS software (version 9.1.3; SAS Institute, Inc., Cary, NC, USA) was used. The statistical tests used in this study were counseled by the Seoul National University Medical Research Collaborating Center (2014-0162).

\section{RESULTS}

\section{Characteristics of primary nonmalignant spine tumor}

The overall incidence rate of primary nonmalignant spine tumors was 2.35 per 100000 persons during the study period. The primary nonmalignant spine tumor incidence rates among the 6 tested age groups ranged from 0.76 to 4.54 per 100000 persons in the group 60-69 years of age which had the highest incidence rate of a primary nonmalignant spine tumor. Moreover, the incidence rate of a primary nonmalignant spine tumor increased with age $(p \leq 0.001$, Table 1$)$.

The overall primary nonmalignant spine tumor incidence rate for male patients was 2.08 per 100000 persons, whereas female patients had a significantly higher overall incidence rate of 2.62 per 100000 persons ( $p=0.001$, Table 1 ). The annual incidence rate of primary nonmalignant spine tumors increased significantly from 2009 to 2011 ( $p=0.012$, Table 1).

The incidence rates of primary nonmalignant spine tumors were 0.22 per 100000 persons with a tumor in vertebral column, 0.01 per 100000 in the pelvis \& sacrum \& coccyx, 0.54 per 100000 in the spinal meninges, 1.58 per 100000 in the spinal cord (Table 1).

The overall incidence rate for male patients with a primary nonmalignant spine tumor was significantly lower than that for female patients with a tumor in the spinal meninges $(0.43$ vs. 0.65 per 100000 persons, $p=0.005$ ), and in the spinal cord (1.45 vs. 1.71 per 100000 persons, $p=0.04$ ).

When each case of a primary nonmalignant spine tumor was stratified by patient age at diagnosis, the group over 70 years of age had the highest incidence rate of a tumor in the vertebral column and the pelvis \& sacrum \& coccyx. In contrast, the group 60-69 years of age had the highest incidence rate in the spinal meninges and spinal cord. In every primary nonmalignant spine tumor, the incidence rate increased with age ( $p \leq 0.001,0.008,<0.001$, and $<0.001$, respectively).

The annual primary nonmalignant spine tumor incidence rate increased significantly from 2009 to 2011 in patients with vertebral column, spinal meninges, and spinal cord tumors ( $p=0.019,0.041$, and 0.035 , respectively; Table 1).

\section{Characteristics of primary malignant spine tumor}

The overall incidence rate for a primary malignant spine tumor was 0.70 per 100000 persons during the study period. The primary malignant spine tumor incidence rates among the 6 tested age groups ranged from 0.32 to 1.72 per 100000 persons for the group over 70 years of age, which had the highest incidence rate of a primary malignant spine tumor. Moreover, the primary malignant spine tumor incidence rate increased with age ( $p \leq 0.001$, Table 2$)$.

The overall incidence rate for a primary malignant spine tumor for male patients was 0.80 per 100000 persons, whereas female patients had a significantly lower overall incidence rate of 0.61 per 100000 persons ( $p=0.03$, Table 2 ).

The incidence rates for a primary malignant spine tumor were 0.26 per 100000 persons with a tumor in the vertebral column, 0.04 per 100000 in the pelvis \& sacrum \& coccyx, 
Epidemiology of Primary Spine Tumor | Sohn S, et al.

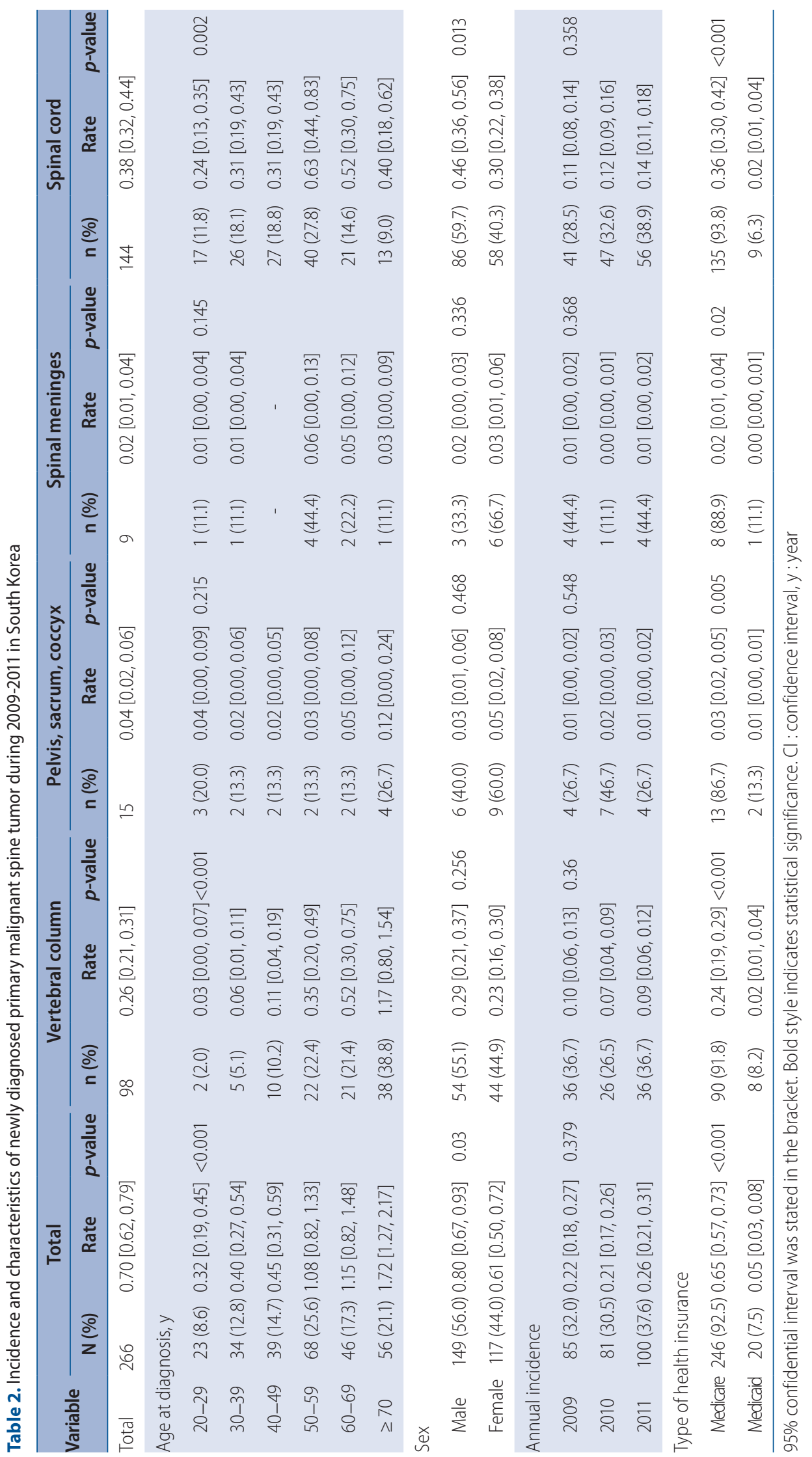


0.02 per 100000 in the spinal meninges, and 0.38 per 100000 persons in the spinal cord (Table 2).

When each case of a primary malignant spine tumor was stratified by patient age at diagnosis, the group over 70 years of age had the highest incidence rate for a tumor in the vertebral column and in the pelvis \& sacrum \& coccyx. In contrast, the group 50-59 years of age had the highest incidence rate for a tumor in the spinal meninges and spinal cord. For tumors in the vertebral column and the spinal cord, the incidence rate increased with age ( $p \leq 0.001$ and $p=0.002$, Table 2$)$.

The overall incidence rate of primary malignant spinal cord tumor in males was significantly higher than that in female patients ( 0.46 vs. 0.30 per 100000 persons, $p=0.013$, Table 2 ).

The annual primary malignant spine tumor incidence rate was not significantly different in any patient group (Table 2).

\section{Annual medical cost and hospital stay duration for those with a primary nonmalignant spine tu- mor}

Total average annual medical costs, including inpatient and outpatient services, ranged from 1627 USD (pelvis \& sacrum \& coccyx tumors) to 6601 USD (spinal cord tumor). With regard to outpatient services, vertebral column tumors were associated with the highest average annual costs $(1476 \pm 1751$ USD), whereas pelvis \& sacrum \& coccyx tumors were associated with the lowest average annual cost (830 \pm 514 USD). Regarding inpatient services, spinal cord tumors were associated with the highest average annual cost (6640 \pm 8590 USD), whereas the pelvis \& sacrum \& coccyx tumors showed the lowest average annual cost ( $797 \pm 575$ USD, Table 3$)$.

The total average annual hospital stay duration, including inpatient and outpatient services, ranged from 46 (pelvis \& sacrum \& coccyx tumors) to 62 (spinal meninges tumor) days. With regard to inpatient services, spinal cord tumors were associated with the longest hospital duration (38 $\pm 63 \mathrm{~d}$ ), whereas pelvis \& sacrum \& coccyx tumors were associated with the shortest hospital stay duration $(11 \pm 11 \mathrm{~d}$, Table 4$)$.

\section{Annual medical cost and hospital stay duration for those with a primary malignant spine tumor}

Total average annual medical costs, including inpatient and outpatient services, ranged from 12137 USD (spinal meninges tumor) to 20825 USD (pelvis \& sacrum \& coccyx tumors). With regard to outpatient services, spinal cord tumors were associated with the highest average annual costs $(4022 \pm 4022$ USD), whereas vertebral column tumors were associated with the lowest average annual cost (2390 \pm 3528 USD). Regarding inpatient services, pelvis \& sacrum \& coccyx tumors were associated with the highest average annual cost $(18737 \pm 18110$ USD), while spinal meninges tumors were associated with the lowest average annual cost (8873 \pm 7037 USD, Table 4).

The total average annual hospital stay duration, including inpatient and outpatient services, ranged from 78 (spinal meninges tumors) to 120 (pelvis \& sacrum \& coccyx tumors)

Table 3. Annual medical cost and hospital stay of newly diagnosed primary nonmalignant spine tumor patients during 2009-2011

\begin{tabular}{llccc}
\hline & \multicolumn{1}{c}{ Type of service } & Patients & Annual cost (USD) & Annual hospital stay (day) \\
\hline Vertebral column & Total & $84(100.0)$ & $5984 \pm 5485$ & $57 \pm 45$ \\
& Inpatient service & $70(83.3)$ & $5410 \pm 4626$ & $29 \pm 34$ \\
& Outpatient service & $84(100.0)$ & $1476 \pm 1751$ & $33 \pm 28$ \\
Pelvis, sacrum, coccyx & Total & $3(100.0)$ & $1627 \pm 65$ & $46 \pm 8$ \\
& Inpatient service & $3(100.0)$ & $797 \pm 575$ & $11 \pm 11$ \\
Spinal meninges & Outpatient service & $3(100.0)$ & $830 \pm 514$ & $35 \pm 18$ \\
& Total & $205(100.0)$ & $6109 \pm 6026$ & $62 \pm 64$ \\
Spinal cord & Inpatient service & $176(85.9)$ & $5610 \pm 5407$ & $37 \pm 58$ \\
& Outpatient service & $204(99.5)$ & $644 \pm 9606$ & $31 \pm 34$ \\
& Total & $596(100.0)$ & $6601 \pm 8452$ & $38 \pm 63$ \\
\hline
\end{tabular}

Values are presented as number (\%) or mean \pm standard deviation. USD : US dollar 
Table 4. Annual medical cost and hospital stay of newly diagnosed primary malignant spine tumor patients during 2009-2011

\begin{tabular}{llccc}
\hline & \multicolumn{1}{c}{ Type of service } & Patients & Annual cost (USD) & Annual hospital stay (day) \\
\hline Vertebral column & Total & $98(100.0)$ & $14183 \pm 12616$ & $84 \pm 68$ \\
& Inpatient service & $89(90.8)$ & $12635 \pm 11443$ & $59 \pm 55$ \\
& Outpatient service & $96(98.0)$ & $2390 \pm 3528$ & $30 \pm 40$ \\
Pelvis, sacrum, coccyx & Total & $15(100.0)$ & $20825 \pm 19652$ & $120 \pm 85$ \\
& Inpatient service & $14(93.3)$ & $18737 \pm 18110$ & $98 \pm 80$ \\
& Outpatient service & $15(100.0)$ & $3338 \pm 5782$ & $29 \pm 16$ \\
Spinal meninges & Total & $9(100.0)$ & $12137 \pm 8150$ & $78 \pm 48$ \\
& Inpatient service & $9(100.0)$ & $8873 \pm 7037$ & $42 \pm 42$ \\
Spinal cord & Outpatient service & $9(100.0)$ & $3264 \pm 5111$ & $36 \pm 22$ \\
& Total & $143(100.0)$ & $15723 \pm 15723$ & $100 \pm 95$ \\
& Inpatient service & $124(86.7)$ & $14673 \pm 14673$ & $78 \pm 95$ \\
\hline
\end{tabular}

Values are presented as number (\%) or mean \pm standard deviation. USD : US dollar

days. With regard to inpatient services, pelvis \& sacrum \& coccyx tumors were associated with the longest hospital duration $(98 \pm 80 \mathrm{~d})$, whereas spinal meninges tumors were associated with the shortest hospital stay duration $(42 \pm 42 \mathrm{~d}$, Table 4).

\section{Survival rates according to each primary malig- nant spine tumor}

Actuarial overall survival rates in primary malignant spine tumors were $88.2 \%, 76.5 \%$, and $71.8 \%$ at 3,12 , and 24 months, respectively (Fig. 1). For malignant vertebral column tumors, overall survival rates were $75.0 \%, 61.1 \%$, and $55.6 \%$ at 3,12 , and 24 months, respectively. For malignant pelvis \& sacrum \& coccyx tumors, corresponding overall survival rates were $100.0 \%, 50.0 \%$, and $50.0 \%$ at 3,12 , and 24 months. For malignant spinal meninges tumors, overall survival rates were $100.0 \%, 100.0 \%$, and $100.0 \%$ at 3,12 , and 24 months, respectively. For malignant spinal cord tumors, the corresponding overall survival rates were $97.6 \%, 90.2 \%$, and $85.4 \%$ at 3, 12, and 24 months (Fig. 2). When we assume median survival periods, the overall median survival duration for those with primary malignant spine tumors was $1162 \mathrm{~d}$ (38.73 months). Estimated median overall survival periods for malignant vertebral column, malignant pelvis \& sacrum \& coccyx, malignant spinal meninges, and malignant spinal cord tumors were 1106 days (36.87 months), 652 days (21.73 months), 1292 days (43.07 months), and 1218 days (40.6

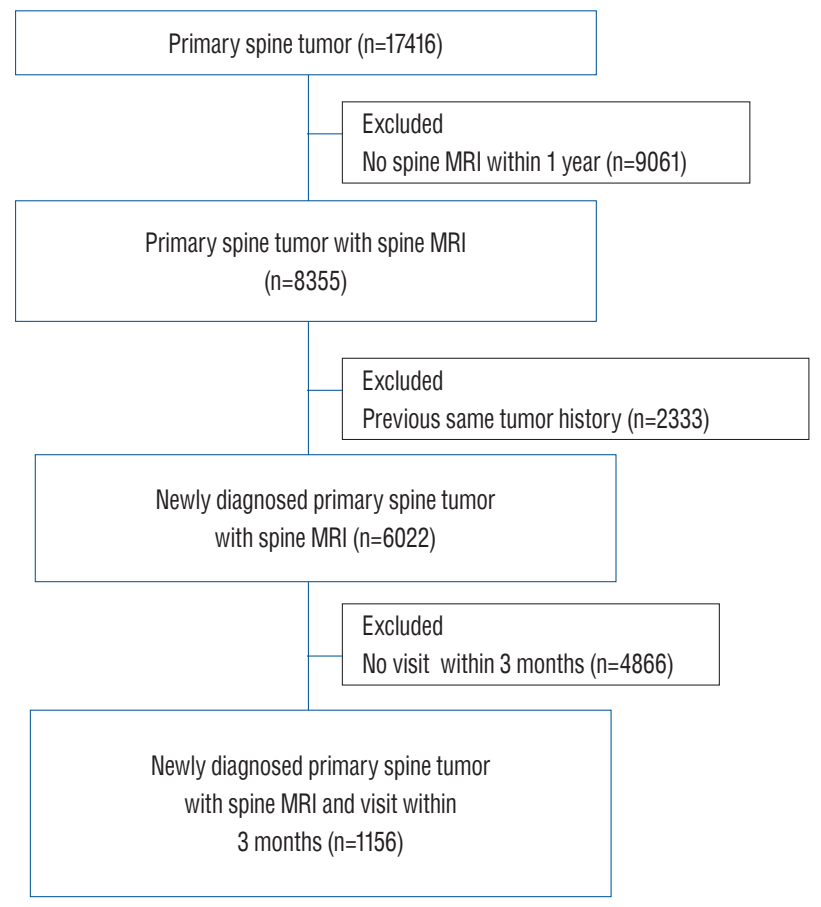

Fig. 1. Flow diagram of screened patients in this study. MRI : magnetic resonance imaging.

months), respectively (Fig. 3).

Cox regression model results showed that male sex, medicare insurance were significantly positive factors for those with a primary malignant spine tumor (Table 5). 


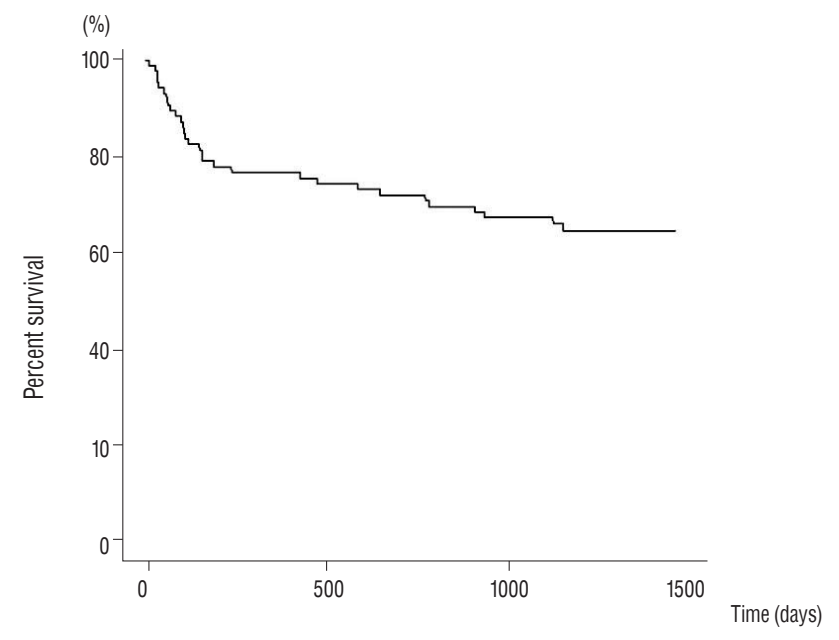

Fig. 2. Kaplan-Meier estimate of overall survival in newly diagnosed primary malignant spine tumor patients.

Table 5. Risk of death in patients with primary malignant spine tumor, 2009-2011

\begin{tabular}{llcc}
\hline \multicolumn{1}{c}{ Characteristics } & HR & 95\% Cl & $\boldsymbol{p}$-value \\
\hline Age & 1.013 & $0.983-1.044$ & 0.3911 \\
Female & 2.819 & $1.051-7.564$ & 0.0396 \\
Medicaid & 4.086 & $1.525-10.944$ & 0.0051 \\
CCl & 1.125 & $0.94-1.347$ & 0.1999 \\
\hline Primary malignant spine tumor & 0.806 & $0.108-6.029$ & 0.8339 \\
\hline
\end{tabular}

Bold style indicates statistical significance. $\mathrm{HR}$ : hazard ratio, $\mathrm{Cl}$ : confidence interval, $\mathrm{CCl}$ : Charlson comorbidity index

\section{DISCUSSION}

In this study, we provided the characteristics, annual medical cost, annual hospital stay duration, and survival rate according to each type of primary spine tumor. This study offers a more detailed analysis than that in our previous descriptive nation-wide spine tumor study ${ }^{17)}$.

\section{Characteristics of primary nonmalignant and malignant spine tumors}

The most common type of primary malignant and primary nonmalignant spine tumors was a neoplasm of the spinal cord. The second most common tumor type was a neoplasm of the vertebral column among the primary malignant tumors and neoplasm of the spinal meninges among the primary nonmalignant spine tumors. This tendency is in good

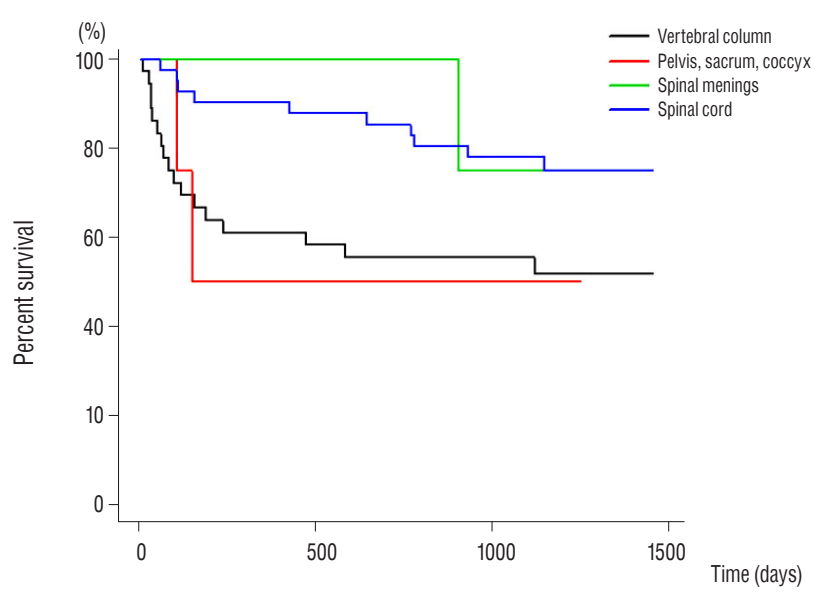

Fig. 3 . Kaplan-Meier estimates of overall survival associated with each newly diagnosed primary malignant spine tumor patients.

agreement with a histology based nationwide Korean study ${ }^{7}$. As we described in our previous study, this tendency is in line with previous studies which found that spinal cord tumors were the most common, followed by spinal meninges tumors ${ }^{3,15)}$. On the other hand, the present findings contrast with those of earlier studies in which spinal meninges tumors were found to be as prevalent as or more prevalent than spinal cord tumors ${ }^{6,13)}$.

Incidence of primary nonmalignant spine tumor was higher in women than that in men (Table 1). On the other hand, incidence of primary malignant spine tumor was lower in women than that in men (Table 2). This tendency was similar to previous a recent Korean study ${ }^{7)}$ and previous studies of the United States $^{3,15)}$.

Age-specific incidence patterns differ between malignant and non-malignant primary spine tumors. For primary nonmalignant spine tumors, incidence rates peaked from 60 to 69 years of age and declined thereafter. However, for primary malignant spine tumors, incidence rates gradually increased with age. These patterns were identical to those in a previous histology based population study in Korea ${ }^{7}$ and can also be found in studies focusing on Estonia and the United States ${ }^{3,10}$.

The annual primary nonmalignant spine tumor incidence rate increased significantly from 2009 to 2011, however, the annual primary malignant spine tumor incidence rate did not change significantly from 2009 to 2011. This result may differ slightly a recent Norwegian population-based study that the incidence rate of primary intraspinal tumors reached a plateau after $2002^{19}$. Although this observation is difficult to explain, 
it may be due to an increase in diagnostic rates, which in turn may be partly attributable to an increase in the overall willingness to be examined as a result of the decrease in the patients' burden of the medical cost from $10 \%$ to $5 \%$, which has been in effect since 2010 .

\section{Annual medical cost and hospital stay duration for those with primary nonmalignant and malig- nant spine tumors}

A recent intramedullary spine tumor study of a US population reported that the mean hospital medical cost ranged from 45452.24 USD in 2003 to 76698.96 USD in $2010^{16)}$. While an intramedullary spine tumor cannot be considered to be similar to all primary spinal cord tumors, the medical costs associated with a spinal cord tumor in the US are higher than in South Korea according to our study (6640 USD for a primary nonmalignant spine tumor and 14673 USD for a primary malignant spine tumor). The medical cost in our study was the annual medical cost while the medical cost in the US study included hospital stay after surgery. The mean hospital stay in the US was around 7 days $^{16)}$. The mean annual hospital stay was 38 days in our study. When we consider the hospital stay duration, the medical cost of a primary spine tumor in Korea may be lower than that in the US.

\section{Survival rate according to each type of primary malignant spine tumor}

In this study, we estimated the median survival period of each type of primary malignant spine tumor. For vertebral column and pelvis \& sacrum \& coccyx tumors, the median survival periods were 1106 days (36.87 months), 652 days (21.73 months), respectively. These survival periods were similar to those in a recent study based on a US population which found a survival period for patients with malignant primary osseous spinal neoplasms of 11 to 50 months ${ }^{14}$.

There have been several studies of the prognostic factors which affect patients with primary spine tumors ${ }^{14,18}$. In our study, male sex and medicare insurance were significantly positive factors affecting survival. The tumor type and age of the patient were not significant factors affecting survival. These results differ somewhat from those in previous reports which found that each tumor location and/or age were prognostic factors ${ }^{14,18}$. These differences may be attributed to the small sample sizes and to population differences. This ob- servation is hard to explain because we cannot obtain each patient's personal information. In primary malignant spine tumor, the incidence of male patient is much higher than that of female patients ( $\mathrm{n}=149$ vs. 117). However, bone tumor such as vertebral column or pelvis (sacrum and coccyx) was relatively prevalent in female patient ( $\mathrm{n}=54$ vs. $44, \mathrm{n}=6$ vs. 9).When we consider median overall survival periods of malignant bone tumor such as osteosarcoma or Ewing sarcoma are less than 5 years ${ }^{1)}$, we hypothesize that histological different prevalence may affect survival difference on female patients. Female cancer patients and Medicaid patients are less prone to undergo aggressive treatment which may affect survival period. However, further studies are warranted to analyze prognostic factors affecting survival of those with a primary malignant spine tumor.

\section{Limitations}

Several limitations of this study should be noted. First, as this is a nationwide study, there may be regional variations with regard to disease diagnosis, coding, and reporting. The researchers using the HIRA database could not validate the individual registrations, since we are only able to access de-identified database, which is mandated by the law for protection of personal information. In addition, there may be differences between the data in the HIRA database and those in the histology based cancer registry. However, we verified that our database was not greatly different from a previous histology based report $\mathrm{t}^{7}$. Second, other conditions such as neurologic deficits and the histologic grade of the tumor could not be analyzed due to a lack of information in the HIRA database. Third, the presence of additional conditions is inevitable when creating research definitions for newly diagnosed primary spine tumor and death. However, as described in our previous study ${ }^{17)}$, we investigated the influence of other limiting conditions by comparing the results with hospital record data obtained from a tertiary hospital. In addition, although a multidisciplinary panel consisting of spine surgeons, epidemiologists, and a radiation oncologist reached consensus on a definition of death, we also compared data from several other durations and verified that the results were similar. Nevertheless, with all of these limitations in mind, this is the first nationwide study to analyze hospital utilization of each tumor type and survival as they pertain to primary spine tumors in Asia. 


\section{CONCLUSION}

This is a nationwide study of newly diagnosed primary spine tumors in Korea. Our study provides a detailed investigation of the characteristics, medical utilization, and survival rates of patients newly diagnosed with primary spine tumors in Korea.

The incidence rate of a diagnosis of a primary spine tumor was found to increase with age, and diagnosis year. Medical costs in Korea associated with this condition may be lower than those in the US. Male sex and medicare insurance were significantly positive factors affecting the survival rates of those with primary malignant spine tumors.

\section{- Acknowledgments}

This work was supported by the National Evidence-based Healthcare Collaborating Agency in South Korea (No. NECAA-13-006). The funding sources had no role in study design, data collection, analysis, manuscript preparation, or the decision to submit the manuscript.

\section{References}

1. Arshi A, Sharim J, Park DY, Park HY, Yazdanshenas H, Bernthal NM, et al. : Prognostic determinants and treatment outcomes analysis of osteosarcoma and Ewing sarcoma of the spine. Spine J, 2016 [Epub ahead of print]

2. Chi JG, Khang SK : Central nervous system tumors among Koreans--a statistical study on 697 cases. J Korean Med Sci 4 : 77-90, 1989

3. Duong LM, McCarthy BJ, McLendon RE, Dolecek TA, Kruchko C, Douglas $\mathrm{LL}$, et al. : Descriptive epidemiology of malignant and nonmalignant primary spinal cord, spinal meninges, and cauda equina tumors, United States, 2004-2007. Cancer 118 : 4220-4227, 2012

4. Elia-Pasquet $S$, Provost $D$, Jaffre $A$, Loiseau $H$, Vital $A$, Kantor $G$, et al. : Incidence of central nervous system tumors in Gironde, France. Neuroepidemiology 23 : 110-117, 2004

5. Engelhard HH, Villano JL, Porter KR, Stewart AK, Barua M, Barker FG, et al. : Clinical presentation, histology, and treatment in 430 patients with primary tumors of the spinal cord, spinal meninges, or cauda equina. J Neurosurg Spine $13: 67-77,2010$

6. Johannesen TB, Angell-Andersen E, Tretli S, Langmark F, Lote K : Trends in incidence of brain and central nervous system tumors in Norway,
1970-1999. Neuroepidemiology 23 : 101-109, 2004

7. Jung KW, Park KH, Ha J, Lee $\mathrm{SH}$, Won YJ, Yoo H : Incidence of primary spinal cord, spinal meninges, and cauda equina tumors in Korea, 20062010. Cancer Res Treat 47 : 166-172, 2015

8. Kim CH, Chung CK, Park CS, Choi B, Kim MJ, Park BJ : Reoperation rate after surgery for lumbar herniated intervertebral disc disease : nationwide cohort study. Spine (Phila Pa 1976) 38 : 581-590, 2013

9. Lee YH, Yoon SJ, Kim EJ, Kim YA, Seo HY, Oh IH : Economic burden of asthma in Korea. Allergy Asthma Proc 32 : 35-40, 2011

10. Liigant A, Asser T, Kulla A, Kaasik AE. Epidemiology of primary central nervous system tumors in Estonia. Neuroepidemiology 19 : 300-311, 2000

11. Lim SJ, Kim HJ, Nam CM, Chang HS, Jang YH, Kim S, et al. : Socioeconomic costs of stroke in Korea : estimated from the Korea national health insurance claims database. J Prev Med Public Health 42 : 251-260, 2009

12. Martin BI, Mirza SK, Comstock BA, Gray DT, Kreuter W, Deyo RA : Reoperation rates following lumbar spine surgery and the influence of spinal fusion procedures. Spine (Phila Pa 1976) 32 : 382-387, 2007

13. Materljan E, Materljan B, Sepcic J, Tuskan-Mohar L, Zamolo G, ErmanBaldini I : Epidemiology of central nervous system tumors in Labin area, Croatia, 1974-2001. Croat Med J 45 : 206-212, 2004

14. Mukherjee D, Chaichana KL, Gokaslan ZL, Aaronson O, Cheng JS, McGirt MJ : Survival of patients with malignant primary osseous spinal neoplasms : results from the Surveillance, Epidemiology, and End Results (SEER) database from 1973 to 2003. J Neurosurg Spine 14 : 143-150, 2011

15. Schellinger KA, Propp JM, Villano JL, McCarthy BJ : Descriptive epidemiology of primary spinal cord tumors. J Neurooncol 87 : 173-179, 2008

16. Sharma M, Sonig A, Ambekar S, Nanda A : Discharge dispositions, complications, and costs of hospitalization in spinal cord tumor surgery : analysis of data from the United States Nationwide Inpatient Sample, 2003-2010. J Neurosurg Spine 20 : 125-141, 2014

17. Sohn S, Kim J, Chung CK, Lee NR, Park E, Chang UK, et al. : Nationwide epidemiology and healthcare utilization of spine tumor patients in the adult Korean population, 2009-2012. Neuro Oncol Pract 2 : 93-100, 2015

18. Szoverfi Z, Lazary A, Bozsodi A, Klemencsics I, Eltes PE, Varga PP : Primary Spinal Tumor Mortality Score (PSTMS) : a novel scoring system for predicting poor survival. Spine J 14 : 2691-2700, 2014

19. Weber C, Gulati S, Jakola AS, Habiba S, Nygaard OP, Johannesen TB, et al. : Incidence rates and surgery of primary intraspinal tumors in the era of modern neuroimaging : a national population-based study. Spine (Phila Pa 1976) 39 : E967-E973, 2014

20. Yoon YK, Park GC, An H, Chun BC, Sohn JW, Kim MJ : Trends of antibiotic consumption in Korea according to National Reimbursement Data (2008-2012) : a population-based epidemiologic study. Medicine (Baltimore) 94 : e2100, 2015 